\title{
La valoración del paisaje cultural como instrumento de intervención territorial
}

\author{
JUAN CARLOS ETULAIN Y ISABEL LÓPEZ (1)
}

\begin{abstract}
RESUMEN
El artículo presenta una perspectiva del ordenamiento entendiendo al territorio como Paisaje Cultural, producto del binomio naturalezasociedad, y también como una estrategia de política de valoración del territorio como patrimonio, buscando y encontrando identidad. Se pone énfasis en la necesidad de un abordaje multiescalar y se presentan los principales instrumento de intervención territorial (mecanismo institucional, de gestión, técnico, de análisis e intervención).

Palabras-claves: Ordenamiento, escalas, observatorio, carta, catálogo, parque patrimonial.
\end{abstract}

\section{ABSTRACT}

The valuation of the cultural landscape like instrument of territorial intervention

The article presents an overview of the system by understanding the territory as a cultural landscape, a product of the binomial naturesociety, and also as a political strategy of the territory as a heritage assessment, seeking and finding identity. It emphasizes the need for a multiscale approach and presents the main instrument of territorial intervention (institutional arrangement, management, technical, analysis and intervention).

Keywords: Ordering, scales, monitoring, chart, catalog, heritage park.

"Toda nueva intervención -paisajística - sobre un espacio o territorio depende obligatoriamente de los condicionantes y circunstancias, ya sean de orden histórico, político, social, espacial y en última instancia cultural...... ello obliga a afrontar los problemas de ordenamiento y diseño bajo dos ángulos, el del respeto y el de la ambición. Respeto por aquello que valoramos como parte del patrimonio -tangible y/o intangible $-y$ ambición por dar una respuesta que mejore la calidad de vida y avance también en la construcción de la cultura paisajista".

Jacques Simón (ROSELL, 1997).

\section{EL TERRITORIO VISTO DESDE LA PERSPECTIVA DE LOS PAISAJES CULTURALES}

La cuestión del paisaje, el paisajismo y/o la arquitectura del paisaje está en estos comienzos del siglo XXI conquistando y penetrando desde muchos ángulos las ideas y las prácticas, en el campo de la profesión, la formación, la gestión y la investigación.

Tal vez más que en otros momentos, entre los múltiples valores que se le otorgan al paisaje es indiscutible el de constituir un valor histórico cultural que activa significados existenciales y que por lo tanto articulan «ser» y «tiempo», integrando formas de identidad y pertenencia en el sentir de los habitantes y de quienes lo miran. También forma parte de la institucionalidad con que se reconocen los recursos (NAVARRO BELLO, 2004). Por lo tanto, no se discute ya sobre su puesta en valor como derecho y como objeto de análisis. Vale también, hacer paisaje o hacer arquitectura de paisaje y/o paisajismo tanto para mejorarlo, protegerlo y/o recrear uno nuevo.

Desde esta perspectiva, la ciudad y el territorio debe ser una oferta global, no una serie de enclaves en medio de un paisaje degradado y/o banal excluido (BORJA, 2003). Esta compuesto por realidades contradictorias y complejas. Contradictorias como los fenómenos de centro y periferia, así como las dinámicas en curso como la densificación y dispersión. Además en este marco ¿qué valor toman los espacios abiertos de todo tipo?, ¿que políticas territoriales y urbanas deben buscar compromisos entre estas contradicciones mediante una intervención transformadora y permanente sobre realidades múltiples de gran complejidad social y cultural, con actividades diversas y contrapuestas?

El desafío del paisaje hoy es también, como en casos anteriores, la respuesta que puede darse a la región metropolitana, un factor cualificante de la ciudad, de las periferias difusas, de los ejes longitudinales que se pierden en

(1) CIUyT-FAU-UNLP. Calle 47 nº 162, La Plata (1900) Argentina - jcetulain@netverk.com.ar - ilopez.arq@gmail.com. 
"espacios medios" asignificantes.

Aplicar ésta perspectiva permitirá crecer la demanda de relaciones de proximidad, de intercambio entre personas y actividades distintas, de valorización de los sentidos, el tacto, el olor, la voz y la piel en directo (ASCHER, 2004). "El paisaje hoy en muchos espacios no existe, es decir, no existe como paisaje ciudadano. Pero su posibilidad sí. Puede responder a una demanda específica de cualidad del entorno, de identidad, de valorización del territorio pero también contribuir a resolver otro problema, el del desarrollo sostenible" (BORJA, 2003).

En este marco surge el concepto de Paisaje Cultural como una estrategia de política de valoración del territorio como patrimonio, buscando y encontrando identidad, y por lo tanto tratar el ordenamiento como el «ordenamiento del paisaje». Para Sauer (SABATÉ BEL, 2004), el Paisaje Cultural es el resultado de la acción de un grupo social sobre el paisaje natural. La cultura es el agente, lo natural, el medio; el paisaje cultural es el resultado.

Concebir la ciudad y el territorio como producto cultural permite analizarlos como resultado de las actuaciones sociales y políticas además de aplicar sobre ellos una mirada integradora. Por ello, se entiende por paisaje cultural al conjunto de acciones de transformación que la labor humana introduce a lo largo del tiempo en un territorio determinado. Operaciones que, de la mano de los propios actores de ese proceso, van constituyendo el verdadero patrimonio histórico, ambiental y ecológico en que se desarrolla la vida de una comunidad (MARTÍNEZ DE SAN VICENTE, 2005). Paisaje y territorio son dos conceptos y dos hechos indisociables aunque no sinónimos, que importa distinguirlos y saber relacionarlos. El paisaje contribuye a la formación de las culturas locales y es un componente fundamental del patrimonio natural y cultural, aporta al bienestar de los seres humanos y a la consolidación de la identidad. Además, del papel que desempeña en el campo cultural, lo hace en el ecológico, ambiental y social, y como hoy no hay un modelo de ordenamiento urbano y territorial dominante, los enfoques pueden variar de acuerdo al valor que se le otorgue a cada sector del territorio y a como estos se articulan entre sí.

Los Paisajes Culturales están teniendo una creciente importancia en el desarrollo económico regional de base local. La mayor parte de los planes de ordenamiento del siglo XX hicieron hincapié en la dinámica poblacional y en el desarrollo industrial, y utilizaron la zonificación y el proyecto de grandes infraestructuras como instrumentos fundamentales. Hoy en cambio algunas propuestas de ordenamiento territorial de interés empiezan a atender a un nuevo binomio: naturaleza y cultura como partes de un concepto único, patrimonio. Y los Paisajes Culturales pueden constituir un vehículo para alcanzar el objetivo de construir entornos más diversos y cargados de identidad.

La selección de diversas áreas, en base a criterios arquitectónicos, históricos o arqueológicos incorporando a veces ciudades enteras y paisajes extensos, la definición de proyectos estratégicos en las mismas; la voluntad de trabajar desde lo local, diseñando procesos de cooperación y consenso; la protección mediante la transformación, superando posiciones conservacionistas, pretende en definitiva fundir la historia cultural, con origen en el pasado, con la planificación, con la voluntad de proyectar el futuro.

Ocuparse del paisaje en las distintas escalas significa que se tienen que cumplir siempre criterios generales de calidad paisajística. Estos pueden clasificarse según las estrategias que se apliquen: de mejora del paisaje; restauración del paisaje; recuperación del paisaje; valoración del paisaje y/o creación de paisaje; teniendo en cuenta los siguientes objetivos específicos:

- Integrar la dimensión " paisaje" en la planificación territorial,

- Conservar y/o mejorar la calidad del paisaje,

- Priorizar la heterogeneidad paisajística,

- Cualificar el espacio público a través a través del diseño del paisaje.

- Proteger y promover el paisaje del patrimonio intangible ligado al paisaje,

- Posibilitar a los ciudadanos la valoración/visualización del paisaje,

- Garantizar la participación de los ciudadanos sobre las decisiones futuras que afecten el paisaje.

Todo lo anterior, sin embargo, deberá responder al manejo sostenible del territorio que aparece como uno de los puntos clave del paradigma ambiental. Para América Latina sería el enfoque más adecuado, donde sociedad, ecología y economía de recursos se articularían con el fin de posibilitar actuaciones que hagan un uso racional de los recursos a la hora de planificar, diseñar y gestionar un proyecto paisajístico.

En este sentido debiéramos orientar nuestros esfuerzos: en situar el paisaje como eje central de los instrumentos y planes de ordenamiento. Paisaje en su más amplio sentido, natural y cultural; paisaje no como resultado acabado de una cultura, sino como realidad continuamente evolutiva; paisaje y territorio no como mero soporte, sino como factor básico de cualquier transformación. Los Paisajes Culturales están llamados a jugar un papel relevante, porque constituyen la expresión de la memoria, de la identidad de una región.

Por lo tanto, luego de conocer e interpretar el territorio, desde visiones que pueden ser diferentes deben establecerse las orientaciones, directrices o lineamientos generales ya que las cuestiones básicas del ordenamiento y el paisaje cultural resultante consisten en saber que acciones políticas y estrategias hay que poner en práctica, con quienes y donde.

\section{ESCALAS E INSTRUMENTOS}

Para analizar el sitio como objeto de proyecto paisajístico y la función social que alojará, es necesario conocer las principales dimensiones que conforman la organización y configuración territorial y de la ciudad en particular. Porque esta última, es actualmente y será a futuro la forma social de ocupación del espacio más importante y la que necesitará del pensamiento y la acción paisajista.

De este modo, las cualidades del recorte territorial -unidad de paisaje - y las ideas que alimentan un proyecto de paisaje tendrán oportunidad de encontrarse y, "las formas de origen natural y las creadas por el hombre se reunirán como esencia 
del goce visual de un diseño paisajístico" (LAURIE, 1983) para que pueda percibirse, utilizarse y disfrutarse, no sólo en el sentido funcional y utilitario, sino también como espacio de placer y disfrute donde los sentidos puedan conmoverse.

En el centro de esta reflexión es necesario situar los fenómenos, los hechos, el mundo real y material, la configuración y organización espacial del territorio y lograr conocerlo desde lo científico con el fin de lograr unidades que faciliten describirlo, interpretarlo y valorarlo como paisaje; para luego intervenir en cada una de ellas con una visión de alcance paisajístico.

Para realizar estas actividades muchos Estados Europeos han creado una entidad de soporte y colaboración con la administración en todas las cuestiones relacionadas con la elaboración, aplicación y gestión de las políticas de paisaje, el Observatorio de Paisaje (instrumento institucional). Estas a través de la elaboración de las Cartas de Paisaje (instrumento de gestión) - instrumentos de consenso de estrategias entre agentes públicos y privados - colaboran en la concertación y la sensibilización de la sociedad en general, en las políticas de paisaje para promover y fomentar la consideración de él, en los diferentes niveles educativos.

Por definición estas cartas contienen los Catálogos de
Paisaje (instrumento técnico de análisis e intervención) que serán rigurosos desde el punto de vista científico y ecológico. Los criterios para su confección son: a) La coherencia del catálogo con respecto a documentos internacionales; b) La visión integral del paisaje; c) La multiplicidad de valores; d) Cumplir con un enfoque metodológico cualitativo; e) Aplicación al conjunto del territorio; f) Aplicabilidad; y g) Participación y/o valoración de la población, que es imprescindible para analizar el paisaje de una manera integrada.

Los catálogos cumplen la función de ser instrumentos para la planificación territorial y también se aplican y pueden constituir el sostén de las campañas de sensibilización sobre la diversidad ecológica; las políticas de conservación de la naturaleza; y documentos que actúan como línea de base para la actuación o punto de partida para la definición de estrategia regionales o locales encaminadas hacia el desarrollo sostenible del territorio. Proporcionan información para la evaluación ambiental estratégica, el estudio de impacto ambiental, el estudio de impacto paisajístico y fundamentalmente desde el punto de vista de una posible intervención de diseño paisajístico, recortan el territorio en unidades de paisaje para luego realizar el proyecto en la escala adecuada hasta llegar a la micro-escala.

\section{CATÁLOGOS}

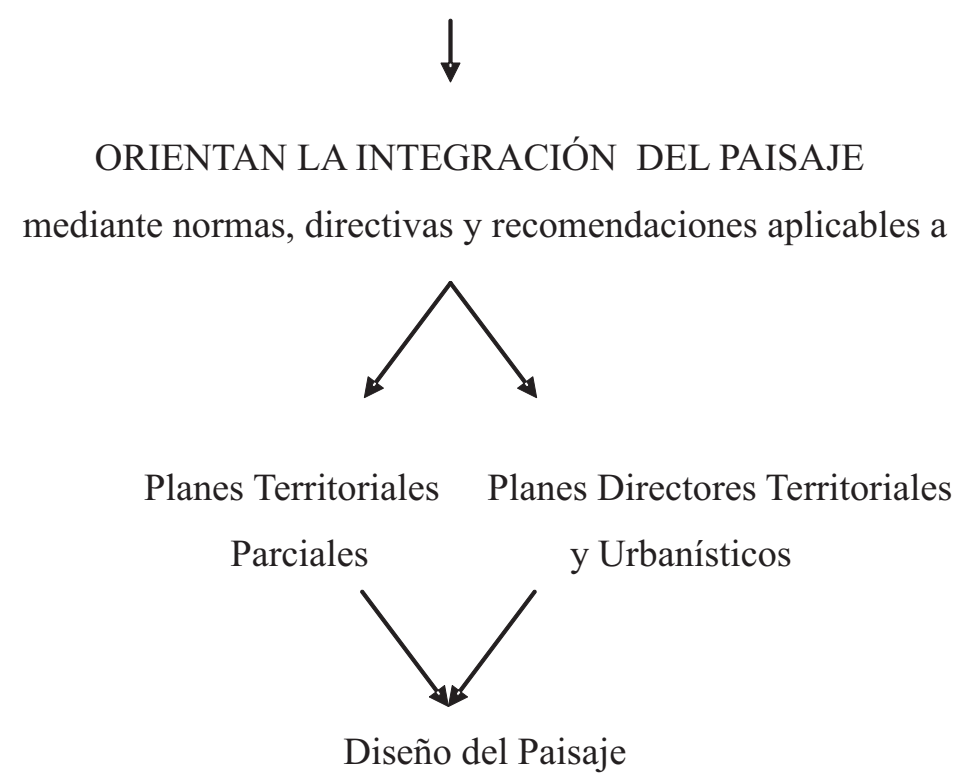

Las unidades de paisaje son porciones del territorio con un mismo carácter. Combinan específicamente componentes paisajísticos de naturaleza ambiental, cultural y estética, de dinámicas claramente reconocibles que le confieren una idiosincrasia diferenciada del resto del territorio. Es decir, que son caracterizadas por un conjunto de variables que hacen un paisaje diferente a otro.

Se basan principalmente en los elementos que estructuran el territorio (montañas, ríos, rutas, etc.) y en su organización (suelo rural, urbano, etc.), considerando también las dinámicas que han contribuido a formar la imagen actual, la tradición

cultural y la historia particular de cada unidad.

El carácter de la unidad dependerá entonces del relieve, de los usos del suelo, de la organización del espacio, de la dimensión histórica, de las transformaciones inmediatas o de las relaciones que se establecen entre la población y su paisaje.

Los criterios generales que se deben seguir para delimitar las unidades de paisaje se relacionan con cubrir todo el territorio; integrar el paisaje en la planificación territorial y definir las unidades de paisaje, basándose en elementos paisajísticos estructurantes. 
Existe también un segundo nivel de unidades de paisaje denominados Paisajes de Atención Especial. Lo componen lugares muy particulares de escasa extensión territorial, que presenten una determinada heterogeneidad, singularidad o complejidad. Se plantearán dentro de estos paisajes objetivos de planeamiento que no sigan el meramente territorial como es el urbanístico.

\section{ANTECEDENTES Y PRÁCTICAS DE INTERVENCIÓN}

Como ya se ha expresado, el paisaje en la inmensa mayoría de los casos es producto no planificado ni proyectado de la actividad humana, pero sí expresa sus valores culturales. "Ninguna concepción estética global presidió su elaboración. La preocupación por la belleza, asociada con alguna función y seguramente a un significado social propio del tiempo y la cultura que lo produjo, solo pudo expresarse en la escala de los edificios o conjuntos edilicios, de los jardines o de los parques" (CLAVAL, 1999).

Sin embargo existe alguna casuística que utilizó y aplicó el campo del conocimiento paisajístico para la creación de algunas ciudades, con técnicas de análisis y valoración del entorno y en el diseño paisajístico, algunas "new towns" en el caso ingles y "nouvelle villes" en el francés, durante la modernidad. También se puede citar, como antecedente, de diseño del paisaje en ámbitos territoriales muy amplios $\mathrm{y}$, como parte de una política regional, la realizada por la Tennesse Valley Administration en Estados Unidos durante la década del '30 del siglo XX y más recientemente.

Desde los ' 70 pero fortalecidas en las últimas dos décadas por la práctica urbanística y el ordenamiento territorial, se llevan a cabo iniciativas y proyectos en los denominados Paisajes Culturales. En 1972, UNESCO celebra una Convención para la protección natural y cultural antecedente de la política de Paisaje Cultural. Luego se inician en varios países las políticas de arqueología industrial y de antropología relacionadas con las cuestiones culturales de la producción del hábitat así como la recuperación de extensos paisajes industriales en EE. UU.

Un instrumento importante para la incorporación de este enfoque en el ordenamiento territorial desde la UNESCO, ha sido Convención Europea del Paisaje realizada en Florencia, Italia. En ella se desarrolla los criterios sobre la protección, ordenación y gestión del paisaje, además de organizar la cooperación europea en estos aspectos. Entra en vigor en marzo del 2004 (29 Estados la han firmado y 16 la han ratificado) incorporando una noción de paisaje como perspectiva integradora y sistémica. Reconoce tres tipos de paisajes: excepcionales, cotidianos y degradados; y define los conceptos de: paisaje, política en materia de paisajes, objetivo de calidad paisajística, protección de los paisajes, gestión de los paisajes, ordenación paisajística (Convención Europea de Paisaje, Florencia, Italia; 2000).

Por otra parte, surgen iniciativas que se fundamentan en el estudio y rehabilitación de elementos patrimoniales y en su utilización para atraer estudiosos y turistas. Surgen los Parques Patrimoniales (instrumento técnico de intervención) como resultado de proyectos que persiguen al tiempo la preservación y revalorización de los recursos patrimoniales del territorio y el desarrollo económico de la región. Como instrumentos conceptuales y también operativos, pueden constituir un vehículo para alcanzar el objetivo de construir entornos más diversos y cargados de significado. Constituyen la expresión de la memoria y de la identidad de una región (SABATÉ BEL, 2004).

El objetivo fundamental suele ser el de integrar, dentro de un estricto respeto a las características de un territorio, preservación, educación, esparcimiento, turismo y desarrollo económico. En muchos casos esto se pretende hacer sentando las bases para una estrecha colaboración entre diferentes administraciones, instituciones y particulares.

La mayor parte de estas iniciativas (50 aproximadamente) están situadas en los Estados Unidos, pero existen muchas y relevantes en Europa. Mediante este instrumento, los recursos patrimoniales (culturales, naturales o mixtos) de los territorios están siendo identificados, protegidos, realzados y promovidos para reforzar las economías locales mediante el aumento del Turismo Cultural, la creación de empleo en servicios y el estudio de nuevas sociedades público-privadas para nuevas oportunidades de inversión. Se reconocen distintos tipos según componente dominante: industriales (Ecomusee de la Communaute Urbaine Le Creusot), infraestructura histórica de transporte (The Baltimore - Ohaio Railroad), fluviales (Blackstone Rive Valle National Heritage Corridor), agrarios (PA Baix Llobregat), militares y arqueológicos (Tennessee Civil war N. Heritage Area) y complejos (IBA Emscher Park) (BUSTAMENTE y PONCE, 2004).

Es un concepto que lleva implícito la noción de proyecto y que como tal, comporta la construcción de una imagen que contribuye a realzar la identidad de un territorio y le provee de elementos que le ayudan a desarrollarse económicamente. Es justamente esta actitud activa, dinámica, proyectual, capaz de incidir e impulsar un territorio determinado, su diferencia con respecto a otro tipo de intervenciones de menor escala o designaciones y nominaciones patrimoniales de carácter más pasivo o estático.

En resumen se puede expresar que el Parque Patrimonial es una entidad compleja que, surge de integrar cuatro necesidades básicas: desarrollo económico, preservación cultural, preservación ambiental (recreación, espacios libres, ocio) y educación e interpretación. Al mismo tiempo, el reconocimiento del parque habría de sentar las bases para una colaboración entre diferentes administraciones, instituciones y el sector privado interesado.

Este instrumento, representa uno de los más importantes avances conceptuales, de importancia teórica para el tratamiento y comprensión integral de los elementos patrimoniales. Con la incorporación de este concepto se superan las visiones pasivas y aisladas de estos elementos, transformándolos en recursos patrimoniales y contribuyendo así a una visión del patrimonio cultural como elemento dinámico. 


\section{REFLEXIÓN FINAL}

Para terminar se puede considerar que el paisaje es un derecho y un recurso que contribuye a consolidar, desde la perspectiva de los Paisajes Culturales, la identidad social e individual de una comunidad, y por esto a su vez puede ser considerado como otra de las dimensiones urbano - territoriales que debe tener proyecto y llevarse a cabo.

Trabajar y pensar en y sobre los conceptos desarrollados en este artículo, podrá colaborar en la extensión y ampliación de la concientización de ésta perspectiva, ya sea para interpretar y valorar el contexto y el lugar de intervención, como para implementar prácticas de diseño, gestión, ordenamiento y protección de los Paisaje Culturales.

"Mejorando y poniendo en valor el paisaje crecerá la demanda de relaciones de proximidad, de intercambio entre personas y actividades distintas, de valorización de los sentidos, el tacto, el olor, la voz y la piel en directo". (ASCHER, 2004).

\section{BIBLIOGRAFÍA CONSULTADA}

ASCHER, F. Los nuevos principios del urbanismo. 1. ed. Madrid: Alianza Editorial, 2004. 93p.

BORJA, J. La ciudad conquistada. 1, ed. Madrid: Alianza Editorial, 2003. 381p.

BUStAMANTE, L.P.; PONCE, C.B. Paisajes Culturales: El Parque Patrimonial como Instrumento de Revalorización y Revitalización del Territorio. Concepción: Departamento de Planificación y Diseño Urbano, Facultad de Arquitectura, Construcción y Diseño, Universidad del Bio-Bio, Comunicación Personal, 2004.

\section{CARTA EUROPEA DE PAISAJE, Florencia, 2000.}

CLAVAL, P. La Geografía Cultural. 1. ed. Buenos Aires: Editorial Eudeba, 1999. 374 p.

ETULAIN, J.C. Los Paisajes Culturales desde la Perspectiva del Patrimonio Mundial. Su correlato empírico en el urbanismo. En: JORNADAS DE INVESTIGACION SI+HCT, 21, y ENCUENTRO REGIONAL DEL CLIP AL TERRITORIO, 3, Buenos Aires. Anales... Secretaria de Investigación de la Facultad de Arquitectura, Diseño y Urbanismo de la UBA. Buenos Aires, 2006, p. 1-10.

LAURIE, M. Introducción a la Arquitectura del Paisaje. 1. ed. Barcelona: Editorial Gustavo Gili, S.A, 1983, 306p. (Colección Arquitectura/Perspectivas).
LÓPEZ, I. La Cultura Paisajista. La Plata: Texto para Maestría en Planeamiento Paisajista y Medio Ambiente. 2008. 9p. (Mimeografiado)

LÓPEZ, I.; ETULAIN, J.C. Políticas Territoriales y Modalidades de Intervención. El Paisaje Cultural en la Región del Gran La Plata: Enfoques Estrategias Instrumentos. La Plata: Proyecto de Investigación del Programa de Incentivos de la Facultad de Arquitectura y Urbanismo de la Universidad Nacional de La Plata. 2006. 31p.

MARTINEZ DE SAN VICENTE, I.; TAMBURRINI, M. C.; BERGALLO N. La Identidad del Territorio como Clave para su Transformación. En: CONGRESO ARQUISUR ARQUITECTURA Y CIUDAD VISIONES CONTEMPORÁNEAS, 5, Tucumán. Anales... Facultad de Arquitectura y Urbanismo, Universidad Nacional de Tucumán. 2006. p. $1-8$

MARTINEZ DE SAN VICENTE, I.; CABRAL, J. Instrumentos de Planeamiento Ordenación del Territorio y Valoración de los Recursos Culturales: Problemáticas, Oportunidades y Desafíos. En: SEMINARIO PAISAJES CULTURALES, PROYECTO EN RED ANPCyT. CIHAM. FADU.UBA, Comunicación Personal, 2005. p. 1-11.

NAVARRO BELLO, G. La importancia del paisaje como patrimonio. Revista Diseño Urbano y Paisaje. Santiago, Centro de Estudios Arquitectónicos, Urbanísticos y del Paisaje Universidad Central de Chile, ano 1, n.2, 2004. p. 41-50.

NUÑEZ, T., LOPO, M. Paisajes Culturales y Planificación Territorial. Una propuesta de gestión y proyecto para el Partido de Tornquist en el Sistema Ventania. Buenos Aires. Documento de Trabajo $N^{*} 8$ de CIHaM. FADU.UBA, Comunicación Personal, 2004. p. 1-19.

ROSELL, Q. Entrevista a Martha Schwartz y Jacques Simon. 2G: Revista Internacional de Arquitectura. Lanscape architecture. Barcelona, v.3, n.3, p. 124-136, 1997.

SABATÉ BEL, J. De la Preservación del Patrimonio a la Ordenación del Paisaje. Revista URBANO, Concepción, Universidad del Bio-Bio, v.7, n. 10, 2004.

ZOIDO NARANJO, F. Principales retos de adaptación de la Convención Europea del Paisaje a las políticas de ordenación del territorio. Sevilla: Facultad de Geografía de la Universidad de Sevilla, 2004. 